Review Article

www.jestr.org

\title{
I Using Porous Material for Heat Transfer Enhancement in Heat Exchangers: Review
}

\author{
M. A. Delavar and M. Azimi* \\ Department of Mechanical Engineering, Babol University of Technology, Babol, IRAN.
}

Received 14 October 2012; Accepted 10 January 2013

\begin{abstract}
The increase in energy cost and energy consumption has required more effective use of energy. The problem of dissipating high heat fluxes has received much attention due to its importance in applications such as heat exchanger. The heat transfer duty of heat exchangers can be improved by heat transfer enhancement techniques. In recent years, Considerable efforts have been made to increase heat transfer rates in heat exchangers by implementing passive enhancement methods that require no direct consumption of external power. On the basis of a theoretical and experimental analysis the conclusion derived was that the best heat transfer enhancement can be reached by the use of porous material as an inexpensive technique to extend the heat transfer area, improve effective thermal conductivity, and mix fluid flow. This paper presents a brief discussion on the application of using porous media to heat exchangers by means of heat transfer enhancement.
\end{abstract}

Keywords: Heat Transfer Enhancement, Heat Exchanger, Porous Media, Porous Heat Exchanger

\section{Introduction}

Effective heat transfer is essential in a variety of energy technologies in order to enable the maximum possible power density and power conversion efficiency needed for economic competitiveness and fuel conservation. The goal of enhanced heat transfer is to encourage or accommodate high heat fluxes. This results in reduction of heat exchanger size, which generally leads to less capital cost [1]. Another advantage is the reduction of temperature driving force, which reduces the entropy generation and increases the second law efficiency. In addition, the heat transfer enhancement enables heat exchangers to operate at smaller velocity, but still achieve the same or even higher heat transfer coefficient [2]. Heat transfer enhancement technology has been widely applied to heat exchanger applications in refrigeration, automobile, process industries etc [3-8].In general, enhanced heat transfer surfaces can be used for three purposes: a- to make heat exchangers more compact in order to reduce their overall volume, and possibly their cost, $b$ - to reduce the pumping power required for a given heat transfer process, or c- to increase the overall UA value of the heat exchanger. Heat transfer enhancement techniques can be divided into two groups: active and passive techniques. The active techniques require external power to facilitate the desired flow modification and the concomitant improvement in the rate of heat transfer. Augmentation of heat transfer by can be achieved by mechanical aids, surface vibration, etc [7]. Passive

*E-mail address: m_r_azimi1991@yahoo.com

ISSN: 1791-2377 @ 2013 Kavala Institute of Technology. All rights reserved.
Techniques do not require any direct input of external power rather they use it from the system itself which ultimately leads to an increase in fluid pressure drop. They generally use surface or geometrical modifications to the flow channel by incorporating inserts or additional devices. They promote higher heat transfer coefficients by disturbing or altering the existing flow behavior except for extended surfaces. So the passive techniques require fluid additives or special surface geometries [9].

In design of compact heat exchangers, passive techniques of heat transfer augmentation can play an important role if a proper passive insert configuration can be selected according to the heat exchanger working condition (both flow and heat transfer conditions).

According to theoretical and experimental analysis [10], the best thermal efficiency can be reached by the use of porous material with a large heat-exchanger surface, a high radial and low axial thermal conductivity (i.e. with a marked anisotropy of thermal conductivity), and a small radius of the heat exchanger operating at lower flows of cooling agent. The present study deals with a heat transfer enhancement method using porous media in heat exchangers. Porous heat exchanger. A Porous medium is a composite medium containing interconnected voids or solid particles embedded into fluid containing medium. Porous media and transport are becoming increasingly important in heat exchanger analysis and design. While the working fluid flows through the porous media, it is heated or cooled by the interaction of the porous walls. The advantage of the porous media as the heat exchanger is its wide contact area to the working fluid. Furthermore, due to its tortuous shape the heat transfer is enhanced. The employment of porous media 
leads to a great increase in heat transfer rate; however, due to the structural features of the porous media, its employment also enlarges the contact area between the porous media and the fluid flow at the same time, thus increases the flow resistance significantly [11].

In work [12] demonstrated that the values of Nusselt number could be increased by as much as 50 percent, for laminar flows in channels with porous material. Moreover, the convective heat transfer coefficient is higher for systems filled with porous material than the systems without porous material due to the high thermal conductivity of the porous matrix compared with the fluid thermal conductivity, especially for gas flows.

Huang, et al. [11] developed and inserted the porous media with a slightly smaller diameter to a tube in the core of the tube under the constant and uniform heat flux condition. The flow resistance and heat transfer characteristics of the air flow for laminar to fully turbulent ranges of Reynolds numbers are investigated experimentally and numerically. There are three different porous media used in the experiments with porosity of $0.951,0.966$ and 0.975 , respectively. The effect of porous radius ratio on the heat transfer performance is studied in numerical simulation. Both numerical and experimental results show that the convective heat transfer is considerably enhanced by the porous inserts.

Alkam and Al-Nimr [13] introduced a method by inserting porous substrates at both sides of the inner tube wall; the thermal performance of a conventional concentric tube heat exchanger can be improved. The improvement was investigated numerically and the results showed that inserting the porous substrate may enhance the effectiveness of the heat exchanger considerably, although it increases the pressure drop within the heat exchanger at the same time.

Steady mixed convection boundary-layer flow past inclined or vertical flat surfaces placed in a porous medium has been investigated by Cheng [14], Merkin[15, 16] and Aly et al. [17]. Cheng [14] obtained similarity solutions for the situation where the free stream velocity and the surface temperature distribution vary according to the same power function of the distance along the surface.

The first efforts of estimating the pressure drop through a porous medium have been made from Darcy [18] based on experimental measurements on the volumetric flow rate and pressure difference across a sand band. In Darcy's experiments, an empirical pressure drop correlation was derived relating the pressure drop per unit length of the porous medium, to the mean velocity of the fluid and the hydraulic conductivity.

For some applications there is no need to fill completely the system with the porous medium, as a partial filling of the porous medium is sufficient. Partial filling has advantage of reducing the pressure drop compared with a system filled completely with porous medium. Moreover, partial filling eliminates contact between the porous material and surface, which decreases heat losses from the porous material to the surface [19]. Yoshida, et al [20] investigated the transient characteristics of heat transfer in porous media. In that work, because of high porosity of the porous media, the conduction heat transfer in the radiative converter was neglected. In the non-radiating gas flow analysis, it was assumed that conduction and convection occurs simultaneously and integral method was used to obtain the net radiative heat flux in the porous matrix.

\section{Metal foam}

The thermal transport characteristics of metal foam have been investigated and some of the published results will be reviewed, mainly focusing on applying metal foams to improve the heat transfer rate. Due to the high surface-area density and strong mixing capability for the fluid, open cell metal foams are now regarded as one of the most promising materials for the manufacture of efficient heat exchangers [21].Mayer et al. [22] observed that metallic porous media offer an effective solution in many heating and cooling applications by the virtue of their inherent properties such as large surface area-to-volume ratio, high permeability and thermal dispersion of the fluid due to the complex flow within pore channels. Pressure loss and convective heat transfer in these metallic structures have been investigated for the above mentioned engineering applications.

Pavel and Mohamad [10] further investigated the enhanced heat transfer effect of metallic porous materials that are inserted into the core of a pipe with a constant and uniform heat flux experimentally and numerically. The results obtained showed that compared with the clear flow case where no porous materials was used, higher heat transfer rates can be achieved using porous inserts whose diameters approach the diameter of the pipe and it is very important to get the accurate physical parameters of a porous material from the experiment for a successful numerical simulation.Boomsma, et. al [23] see that the compressed aluminum foams performed well not only in the heat transfer enhancement, but they also made a significant improvement in the efficiency over several commercially available heat exchangers which operate under nearly identical conditions. The metal foam heat exchangers decreased the thermal resistance by nearly half when compared to currently used heat exchangers designed for the same application. Calmidi and Mahajan [21, 24] conducted a comprehensive experimental and numerical study of forced convection in high porosity aluminum metal foam with variety of porosities and pore densities and using air as the fluid and they showed that using metal-foams can significantly improve the heat transfer in heat exchangers, Boomsma and Poulikakos [25] developed a geometrical effective thermal conductivity model of metal foam based on the idealized three dimensional basic cell geometry of a foam.Venugopal, et. all [26], experimentally studied the potential of a inexpensive metal porous insert developed specifically for augmenting heat transfer from the heated wall of a vertical duct under forced flow conditions. The porous insert used consists of a stack of metallic perforated plates filled inside the duct. The characteristic features of the porous medium model on the hydrodynamic and heat transfer behavior have been investigated. When a high temperature gas flows through a porous metal plate with high porosity, the enthalpy of working gas is effectively transformed to the porous medium via an extremely high heat transfer coefficient and large surface area between the flowing gas and the porous plate, and then a large amount of radiant energy is emitted in upstream direction by the porous plate with strong emissive power. This procedure has been used in high temperature heat exchangers to enhance the performance of these equipments [27].

Mahjoob and Vafai [28] studied the effects of micro structural metal foam properties, such as porosity, pore and fiber diameters, pore density, and relative density, on the heat exchanger performance. 


\section{Conclusions}

Heat exchangers are also very important for energy conservation, conversion, recovery and successful implementation of new energy sources, and wide usage of it involves heat recovery. The potential of porous structures (e.g., metallic foam) in heat exchangers is currently undergoing growing interest due to their large surface area per unit of volume. Recently tremendous works have been conducted on heat transfer enhancement and a large number of techniques for heat transfer enhancement have been developed. This work concerns the investigation on effect of porous media on heat transfer rate in heat exchangers.

\section{References}

1. A.E Bergles, Enhancement of Heat Transfer, Heat Transfer 1978 Proceeding of the 6th International Heat Transfer Conference 6, Hemisphere, Washington, D.C. USA (1978), pp. 89-108.

2. W. M. Kays and A. L. London, Compact Heat Exchangers, The National Press, Palo Alto, CA, 1964.

3. A. L. London and C. K. Ferguson, Test Results of Highperformance Heat Exchanger Surfaces Used in Aircraft Intercoolers and Their Significance for Gas-turbine Regenerator Design, Transactions of the ASME, Vol. 71, pp. 17-26, 1949.

4. Q.T. Zhou, S.Y. Huang, Heat Transfer Enhancement in Boiler and Heat Exchanger, China Water Power Press (1991)

5. Leon Liebenberg, Joshua P. Meyer, In-tube passive heat transfer enhancement in the process industry, Applied Thermal Engineering, Volume 27, Issue 16, 2007, 2713-2726

6. A.M. Jacobi, R.K. Shah, Air-side flow heat transfer in compact heat exchangers: A discussion of enhancement mechanism, Heat Transfer Eng, 19 (4) (1998), pp. 29-41.

7. A. E. Bergles, R. L. Webb and G. H. Junkan, Energy Conservation Via Heat Transfer Enhancement, Energy, Vol. 4, pp. 193-200, 1979.

8. J.R. Thome, Heat transfer augmentation of shell-and-tube heat exchangers for the chemical processing industry, Proceeding 2nd European Thermal Sciences 14th UIT National Heat Transfer Conference 1, Edizioni ETS, Pisa, Italy (1996), pp. 15-26

9. A. Bejan and Peter A. Pfister, Jr., Evaluation of Heat Transfer Augmentation Techniques Based on Their Impact on Entropy Generation, Letters in Heat and Mass Transfer, Vol. 7, pp. 97-106, 1980.

10. B. I. Pavel, A. A. mohammad, an experimental and numerical study on heat trnasfer for gas heat exchangers fitted with porous media, international journal of heat and mass transfer, 47, 2004, pp. 4939-4952

11. Z.F. Huang, A. Nakayama, K. Yang, C. Yang, W. Liu, Enhancing heat transfer in the core flow by using porous medium insert in a tube, International Journal of Heat and Mass Transfer 53 (2010) 1164-1174

12. A.A. Mohamad, Heat transfer enhancements in heat exchangers fitted with porous media Part I: constant wall temperature, International Journal of Thermal Sciences 42 (2003) 385-395.

13. M.K. Alkam, M.A. Al-Nimr, Improving the performance of double-pipe heat exchangers by using porous substrates, Int. J. Heat Mass Transfer 42 (1999) 3609-3618.

14. Cheng, P.: Similarity solutions formixed convection from horizontal impermeable surfaces in saturated porous media. Int. J. Heat Mass Transf. 20, 893-898 (1977)
15. Merkin, J.H.: Mixed convection boundary layer flow on a vertical surface in a saturated porous medium. J. Eng. Math. 14, 301-313 (1980)

16. Merkin, J.H.: On dual solutions occurring in mixed convection in a porous medium. J. Eng. Math. 20, 171-179 (1985)

17. Aly, E.H., Elliott, L., Ingham, D.B.: Mixed convection boundarylayer flow over a vertical surface embedded in a porous medium. Eur. J. Mech. B 22, 529-543 (2003)

18. H. Darcy, Les fontaines publiques de la ville de Dyon. Victor Dalmont, 1856.

19. S.Z. Shuja, B. S. Yilbas, M. Kassas, Flow over blocks in a square cavity: influence of heat flux and porosity on heat transfer rates, Vol.48, Issue.8, 2009, pp.1564-1573

20. Yoshida, H., Yung, J.H., Echigo, R. and Tomimura, T., "Transient characteristics of combined conduction, convection and radiation heat transfer in porous media", International Journal of Heat and Mass Transfer, Vol. 33, No. 5, (1990), 847-857.

21. V.V. Calmidi, R.L. Mahajan, Forced convection in high porosity metal foams, Journal of Heat Transfer vol. 122, pp 557-565, 2000.

22. Barbara Mayer, Weigand Bernhard, Andreas Heselhaus, and Michael Crawford, Heat transfer and pressure drop in a regular porous structure at high Reynolds numbers, Technical report, Zeszyty Naukowe Politechniki Poznanskiej, 2011.

23. K. Boomsma, D. Poulikakos, F. Zwick, Metal foams as compact high performance heat exchangers, Mechanics of Materials 35 (2003) 1161-1176.

24. V.V. Calmidi, R.L. Mahajan, The effective thermal conductivity of high porosity fibrous metal foams, ASME. J. Heat. Transf.vol. 121, pp 466-471, 1999.

25. K. Boomsma, D. Poulikakos, On the effective thermal conductivity of a three-dimensionally structured fluid-saturated metal foam, International Journal of Heat and Mass Transfer.vol 44, pp 827-836, 2001.

26. Venugopal, G. Balaji, C. Venkateshan, S. P. , Experimental study of mixed convection heat transfer in a vertical duct filled with metallic porous structures, International Journal of Thermal Sciences , 2010, vol. 49; issue. 2 , pages 340-348.

27. Echigo, R., "Heat transfer augmentation in hightemperature heat exchangers, in: High Temperature Equipment", Hemisphere, Washington, D.C., U.S.A., (1986), 41-72.

28. Shadi Mahjoob, Kambiz Vafai, A synthesis of fluid and thermal transport models for metal foam heat exchangers, International Journal of Heat and Mass Transfer.vol 51,pp 3701-3711,2008 